\title{
Coronavirus Human Infection Challenge Studies: Assessing Potential Benefits and Risks
}

\author{
Euzebiusz Jamrozik (D) - George S. Heriot • Michael J. Selgelid
}

Received: 18 May 2020 / Accepted: 11 August 2020

(C) Journal of Bioethical Inquiry Pty Ltd. 2020

\begin{abstract}
Human infection challenge studies (HCS) have been proposed as a means to accelerate SARSCoV2 vaccine development and thereby help to mitigate a prolonged global public health crisis. A key criterion for the ethical acceptability of SARS-CoV2 HCS is that potential benefits outweigh risks. Although the assessment of risks and benefits is meant to be a standard part of research ethics review, systematic comparisons are particularly important in the context of SARS-CoV2 HCS in light of the significant potential benefits and harms at stake as well as the need to preserve public trust in research and vaccines. In this paper we explore several considerations that should inform systematic assessment of SARS-CoV-2 HCS. First, we detail key potential benefits of SARS-CoV-2 HCS including, but not limited to, those related to the acceleration of vaccine development. Second, we identify where modelling is needed to inform risk-benefit (and thus ethical) assessments. Modelling will be particularly useful in (i) comparing potential benefits and risks of HCS with those of vaccine field trials under different epidemiological
\end{abstract}

E. Jamrozik $(\bowtie) \cdot$ M. J. Selgelid

Monash Bioethics Centre, Monash University, Melbourne,

Australia

e-mail: zeb.jamrozik@monash.edu

E. Jamrozik

Royal Melbourne Hospital Department of Medicine, University of Melbourne, Melbourne, Australia

G. S. Heriot

School of Public Health and Preventive Medicine, Monash University, Melbourne, Australia conditions and (ii) estimating marginal risks to HCS participants in light of the background probabilities of infection in their local community. We highlight interactions between public health policy and research priorities, including situations in which research ethics assessments may need to strike a balance between competing considerations.

Keywords Infectious disease - Human challenge studies · Controlled human infection model $\cdot$ Research Ethics $\cdot$ Risk $\cdot$ Background risk $\cdot$ Modelling .

Coronavirus $\cdot$ COVID-19

\section{Introduction}

Human infection challenge studies (HCS) - i.e., experiments involving the intentional infection of research participants - have improved scientific understanding and public health responses to multiple infectious diseases. In particular, HCS are a key method of accelerating or improving vaccine development, and HCS results have contributed to the recent licensure or approval of vaccines for cholera and typhoid (Jamrozik and Selgelid 2020a; Tacket et al. 1999; Jin et al. 2017). In response to the COVID-19 pandemic, challenge studies have been proposed as a means of accelerating the development of SARS-CoV-2 vaccines (Eyal et al. 2020; Plotkin and Caplan 2020; Schaefer et al. 2020). Novel research designs, particularly where such studies might be controversial as in the case of SARS-CoV-2 HCS, require especially careful ethical evaluation including rigorous 
risk-benefit assessments as well as timely, thorough, public engagement ( WHO Working Group for Guidance on Human Challenge Studies in COVID-19 2020; Bambery et al. 2015; Hope and McMillan 2004; NHMRC and ARC 2018). In this article, we argue that risk-benefit assessment of SARS-CoV-2 HCS should include thorough consideration of the potential benefits and risks of such studies in comparison to other kinds of studies - and that estimates of relevant benefits and risks should, insofar as possible, be informed by mathematical modelling. Section 1 of this article describes the potential public health benefits of HCS, Section 2 illustrates how these benefits should be systematically evaluated in light of comparisons to vaccine field trials in different epidemic settings, and Section 3 evaluates risks to participants as compared to background risk in different epidemic settings.

\section{Public Health Benefits of Challenge Studies}

Recent academic and public debates surrounding SARS-CoV-2 HCS have largely focused on the potential for HCS to accelerate vaccine development through more rapid demonstration of candidate vaccines' efficacy (or lack thereof) (Eyal et al. 2020; Plotkin and Caplan 2020; Schaefer et al. 2020; Cohen 2020). Widespread use of a safe and effective vaccine for SARS-CoV-2 could have enormous global public health benefits, and the sooner mass vaccination commences the greater these benefits would be. One proposal for the use of challenge studies to accelerate SARS-CoV-2 vaccine development involves replacing a traditional Phase III vaccine efficacy field trial with a challenge study, followed by safety testing in a larger cohort and application for licensure or emergency use authorization for the vaccine (if it is shown, via such studies, to be safe and effective) (Fig. 1, option 2) (Eyal et al. 2020). Additional safety and/or efficacy data would then be collected during and after the introduction of the vaccine into public health use (WHO Working Group for Guidance on Human Challenge Studies in COVID-19 2020).

\section{Public Health Benefits of Testing Multiple Vaccines}

The acceleration of Phase III efficacy testing is not the only way that HCS might plausibly accelerate or improve vaccine development (See Fig. 1 and Table 1). Given that many of the more than 100 SARS-CoV-2 candidate vaccines might ultimately need to be tested in humans, HCS would presumably provide the most efficient way to prioritize (or "select") the most promising vaccine candidates worthy of further investigation in field trials (Fig. 1, option 1). This could accelerate vaccine development, for example by permitting early investment in manufacturing capacity for the most promising candidates based on rapid results from challenge studies. Decisions have already been made to develop production capacity for vaccine candidates that have not yet been tested for efficacy (Gates 2020). By providing early estimates of efficacy, challenge studies would enable some manufacturing decisions to be made sooner, to be ready to increase production earlier than would be possible if such decisions were delayed until large field trials were completed-HCS might also make it more likely that investment in production capacity is not wasted on vaccines with low or zero efficacy and instead allocated to those most likely to be highly efficacious. The potential to compare the efficacy of multiple vaccine candidates in a standardized challenge study design would also obviate multiple parallel or sequential field trials that would plausibly require many months or years, tens of thousands of participants, and enormous financial costs (Jamrozik and Selgelid 2020b).

Direct comparative efficacy testing of multiple vaccines in HCS could also increase the likelihood that a more efficacious vaccine is ultimately deployed, for example, by comparing the first licenced vaccine with other candidates. Without conducting HCS, comparative estimates of vaccine efficacy are extremely difficult to obtain (Lurie et al. 2020), yet even small but statistically significant differences in efficacy (e.g., of 10 per cent) might result in large differences in public health outcomes when vaccines are used by millions of people at substantial risk of infection (see Fig. 1, option 3). Similarly, challenge studies might obviate more complex ethical and study design choices regarding optimal methods for comparing a new vaccine to one with early evidence of efficacy (Eyal and Lipsitch 2020). The fact that a particular vaccine happens to be tested first or has stronger financial and/or political support should not preclude other vaccines being tested, especially where there is reason to think that this might result in a safer or more effective vaccine ultimately being deployed for widespread public health use.

\section{Other Public Health Benefits}

Challenge studies could improve vaccine development in other ways - for example by determining vaccine- 


\section{Uses for challenge studies in Phase III trials}

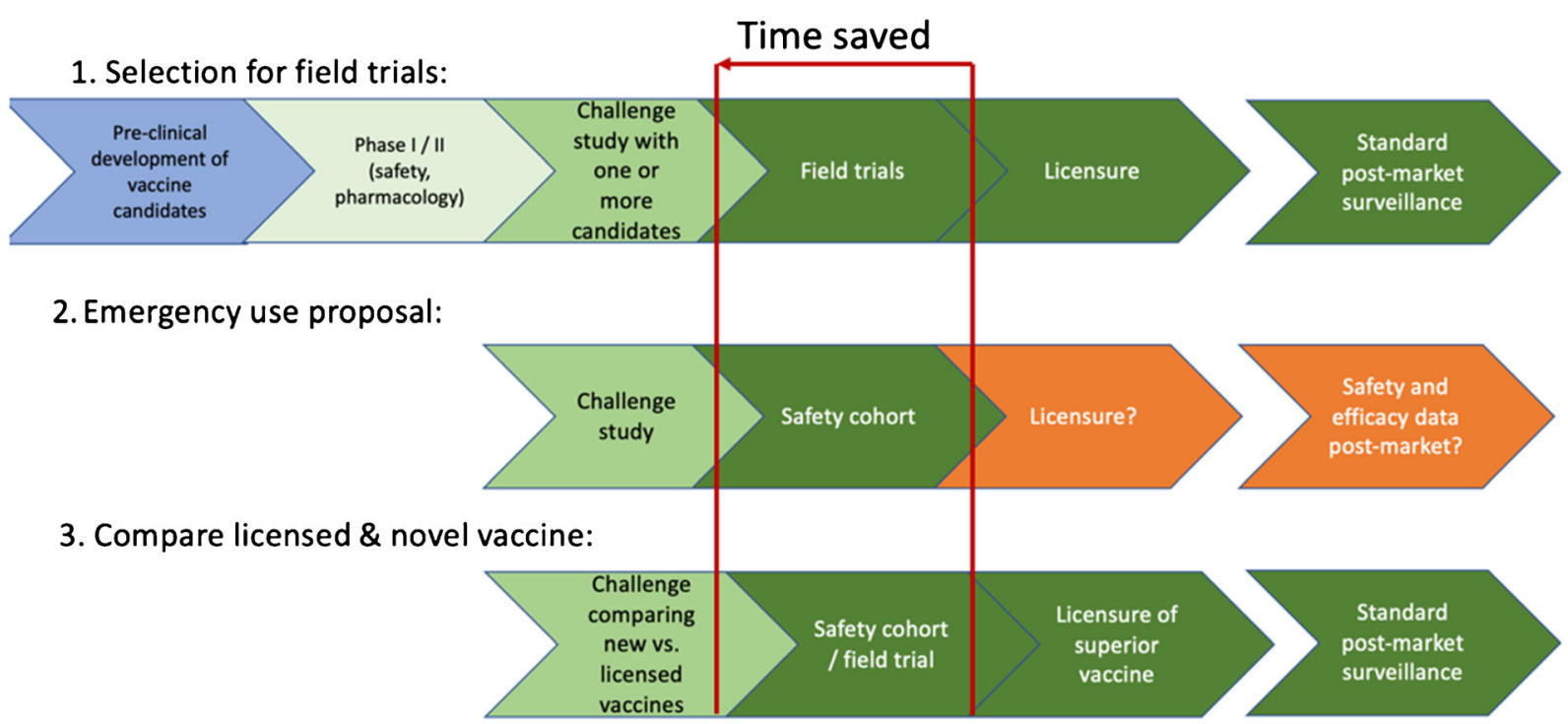

Fig. 1 Uses for challenge studies in Phase III vaccine trials. Challenge studies can be used for Phase III efficacy trials of vaccines in at least three ways. Option 1 describes a standard use: the selection among multiple candidates for the most promising vaccines to enter field trials. Option 2 describes a type of proposal where efficacy data from a challenge study plus safety

related correlates of immune protection that could be used to inform several aspects of vaccine development, including the use of vaccines in populations not involved in efficacy testing (WHO Working Group for Guidance on Human Challenge Studies in COVID-19 2020; Memoli et al. 2016). Immunological correlates of protection can be used in immune bridging studies as a simplified measure to assess whether vaccination of older individuals, children, and pregnant women (who are often excluded from standard efficacy trials) is likely to result in protection against infection. Once correlates have been determined, protection can be measured via the relevant correlate (i.e., immunological blood test), rather than measuring efficacy in terms of reduced rates of infection in a field trial or challenge study. Similarly, HCS provide a unique opportunity to validate the performance of serological assays in predicting protective immunity to infection (Memoli et al. 2016) - more accurate tests would help to inform both future research (Lipsitch et al. 2020) and immediate public health practice (See Table 1) (Phelan 2020). Finally, HCS may provide unique or otherwise difficult to obtain results regarding (i) the characteristics of asymptomatic infection, (ii) the route(s) and risk of transmission during the data from a larger cohort could be submitted in an application for vaccine licensure or emergency use. Whether regulators would accept these data alone, and how this would affect the design of post-market surveillance, remains to be confirmed. Option 3 describes an iterative process where challenge studies could be used to compare a licensed vaccine with a novel vaccine.

early phases of illness (and the effect of different types of protective equipment) (Hilding 1994), and (iii) risks of re-infection after repeat challenge (a study design previously conducted with lower virulence coronaviruses (Callow et al. 1990).

\section{Comparing Challenge Studies to Alternative Research Designs}

The ethical evaluation of a particular challenge study (or programme of studies) should include comparison of the expected benefits and risks with those of other feasible research designs. Since animal challenge studies with SARS-CoV-2 to date do not appear to be highly generalizable to humans (Lurie et al. 2020), the key comparator for HCS would be human vaccine field trials. Vaccine field trials for SARS-CoV-2 might be more difficult (and onerous) than usual because of requirements for intensive testing for asymptomatic infection among participants, although study designs incorporating such testing have been proposed (Kahn et al. 2019). Another key consideration is the effect of local epidemiology on research design and assessment, which we discuss below. 
Table 1 Potential public health benefits associated with COVID19 challenge study designs

\begin{tabular}{|c|c|}
\hline $\begin{array}{l}\text { Aims related to vaccine } \\
\text { development }\end{array}$ & $\begin{array}{l}\text { Examples of public health } \\
\text { benefits }\end{array}$ \\
\hline $\begin{array}{l}\text { 1. Accelerated development of } \\
\text { first vaccine }\end{array}$ & $\begin{array}{l}\text { More cases of infection / disease } \\
\text { prevented by earlier licensure } \\
\text { and deployment of first ef- } \\
\text { fective vaccine }\end{array}$ \\
\hline $\begin{array}{l}\text { 2. Direct comparison of first } \\
\text { licensed vaccine with other } \\
\text { vaccine candidates }\end{array}$ & $\begin{array}{l}\text { Iteration to vaccines with greater } \\
\text { efficacy, resulting in more } \\
\text { cases of infection / disease } \\
\text { prevented by deployment of } \\
\text { superior vaccine }\end{array}$ \\
\hline $\begin{array}{l}\text { 3. Defining vaccine-induced } \\
\text { immune correlates of protec- } \\
\text { tion }\end{array}$ & $\begin{array}{l}\text { Immune bridging studies to } \\
\text { demonstrate vaccine efficacy } \\
\text { in (other) sub-populations }\end{array}$ \\
\hline \multicolumn{2}{|c|}{ Aims related to other scientific knowledge } \\
\hline $\begin{array}{l}\text { 1. Validation of serological tests } \\
\text { of immunity }\end{array}$ & $\begin{array}{l}\text { Reduced disease transmission } \\
\text { by the use of more accurate } \\
\text { tests to inform public health } \\
\text { practice }\end{array}$ \\
\hline $\begin{array}{l}\text { 2. Studying asymptomatic } \\
\text { infection }\end{array}$ & $\begin{array}{l}\text { Refining public health control } \\
\text { measures for asymptomatic } \\
\text { individuals }\end{array}$ \\
\hline $\begin{array}{l}\text { 3. Clarifying transmission } \\
\text { routes and risks }\end{array}$ & $\begin{array}{l}\text { Refining control measures and } \\
\text { use of personal protective } \\
\text { equipment }\end{array}$ \\
\hline $\begin{array}{l}\text { 4. Studying immunity and } \\
\text { re-infection }\end{array}$ & $\begin{array}{l}\text { Refining risk communication } \\
\text { and epidemic models / fore- } \\
\text { casting }\end{array}$ \\
\hline
\end{tabular}

Vaccine Efficacy Trial Design in Different Local Epidemic Contexts

Assessments of potential vaccine research strategies should include direct comparisons between HCS and field trials regarding (i) the time required to produce results estimating vaccine efficacy, (ii) the number of participants involved, and (iii) relevant benefits and risks of research. These comparisons will be complex and may require epidemiological modelling to inform ethical assessments with quantitative data. A higher population incidence of infection, a larger field trial, and a more effective vaccine all allow a field trial to report in a shorter period of time than would otherwise be the case. Conversely, field trials may be infeasible in low incidence populations where public health interventions are successfully suppressing or have eliminated disease transmission - for example, at time of writing (June 2020) field trials would not be feasible in Australia or New Zealand. In such populations, HCS would be the only way to estimate vaccine efficacy (Heriot et al. 2020), yet there are few HCS research centres in Australia and New Zealand and, to our knowledge, none have recently conducted respiratory virus HCS. In some settings, field trial participants might be recruited from higher risk groups, and healthcare workers (HCWs) might be considered candidates for such studies. However, where HCWs have access to adequate protective equipment (and use it appropriately), they may not face significantly higher risks of infection with respiratory viruses than others in the wider community (Bandaranayake et al. 2010). The recruitment of HCWs with poor access to protective equipment without remediating this basic deficiency might be considered unjust and/or exploitative, even though such individuals might benefit from participation in cases where an experimental vaccine turns out to be effective.

Field trials will also be infeasible in inter-epidemic periods. This issue was illustrated by the 2015-16 Zika epidemic: a prominent report on Zika HCS recommended that vaccine efficacy be tested quickly during the epidemic using field trials without the need to expose research participants to challenge infection (Shah et al. 2017). However, by the time vaccines were ready for field trial testing, the epidemic had waned, incidence was low, and herd immunity levels were high in affected communities (as a result of widespread prior infection) - thus making Zika vaccine field trials infeasible (Vannice et al. 2019). Similar conditions will presumably occur in some communities already significantly affected by COVID-19.

The practical difficulty of timing a field trial with an epidemic peak is joined by justice concerns, including that the burdens of field trials would be concentrated in communities already managing a high burden of disease (which may be at least partly attributable to injustice and/or public health policy failures) and also that there would be little community benefit of post-trial access to a vaccine (if shown to be effective in a field trial) due to high levels of herd immunity (Jamrozik and Selgelid 2020b; Wenner 2018). The benefits of any vaccines proven to be safe and effective via field trials would largely accrue to other populations in which public health measures had more successfully controlled disease transmission until such time as an effective vaccine became available.

Elsewhere, we have provided epidemiological models of the conditions under which vaccine field trials could be conducted as quickly as HCS and/or the extent 
to which populations in which field trial research is conducted might stand to benefit from vaccines tested in this way (Heriot et al. 2020). Such models should arguably be used to inform practical decisions about what type(s) of trial(s) could be conducted in particular settings as well as inform ethical judgements about when and where they should ideally be conducted (e.g., relative to local epidemic conditions and public health policies).

\section{Risks to Participants}

It is also important to compare HCS and field trials with respect to the risks to participants. Both HCS and field trials involve exposing participants to the risks related to experimental vaccines including, in some cases, risks of vaccine-enhanced disease (Jamrozik and Selgelid 2020b). On the one hand, HCS involve exposing smaller numbers of participants to such risks and often involve especially close monitoring and early provision of medical care (if required). On the other hand, because of small sample sizes, HCS might fail to detect less common risks of vaccines that could be more easily detected in larger studies such as field trials (Jamrozik and Selgelid 2020b).

Key additional risks of HCS relate to the challenge infection itself. To be ethically acceptable, SARS-CoV2 HCS would need to have multiple risk minimization strategies in place, including (i) selection of low risk participants (e.g., healthy young adults ${ }^{1}$ ), (ii) careful strain selection and development, (iii) careful titration of viral dose, (iv) early diagnosis and availability of all necessary medical care, (v) long-term follow-up of participants, (vi) compensation for any lasting harms, and (vii) stringent infection control including measures to protect and screen research staff for infection (WHO Working Group for Guidance on Human Challenge Studies in COVID-19 2020). Infection with SARS-

\footnotetext{
${ }^{1}$ Where current data provide grounds for reasonable confidence that certain individuals are at significantly higher risk of participation, such individuals should be excluded from participation. There should nonetheless be acknowledgement that even rigorous selection criteria do not preclude there being rare serious outcomes. One potential concern might be the exclusion of certain groups (e.g. ethnic groups) from vaccine research, which might result in the loss of information important for achieving public health benefits for these groups. Such concerns could be remedied by (i) inclusion of lower risk individuals in these groups where such individuals can be confidently identified and/ or (ii) inclusion of such groups in other relevant lower risk research (e.g., immune-bridging vaccine studies).
}

CoV-2 would nevertheless involve residual risks, even for young healthy adults aged 18-30. Risks of hospitalization and fatal infection in this age group (whether healthy or not) have been estimated to be around 0.6-1 per cent and 0.007-0.03 per cent respectively (Verity et al. 2020; Salje et al. 2020). However, significant uncertainty remains, and more data are needed to clarify these risks and determine any risks of lasting harms from SARS-CoV-2 infection (e.g., longer-term lung function deficits, which sometimes occur after other respiratory tract infections (Krzyzanowski et al. 1990)).

While the absolute risk to participants related to the challenge infection is one important consideration, in cases where participants face a background risk of infection (e.g. as a result of a local COVID-19 epidemic), it is also important to assess (via modelling) and consider the marginal risks to participants (i.e., related to the additional probability of being infected as a result of study participation, relative to background risk). The relevance of background risk to judgements regarding risks to participants is sometimes controversial, especially if background risks are attributable to injustice (Jamrozik and Selgelid 2020c). However, background risk has been considered one reason in favour of the ethical acceptability of early challenge studies with yellow fever (Lederer 2008); more recently, it has been considered in favour of Zika HCS (Shah et al. 2017) and HCS in endemic settings more generally - even though, in the case of Zika and (other) diseases endemic to LMICs, one might think that higher incidence of certain infections in particular locations is at least partly due to injustice (Jamrozik and Selgelid 2020a; Lederer 2008).

Marginal risk depends not only on one's probability of being infected and suffering a bad outcome after infection from community transmission (i.e., background risk) but also on the degree to which one might develop immunity as a result of HCS participation (whether from challenge infection or an experimental vaccine) and the probability of having access to a vaccine developed via the participation of others (whether in HCS or field trials). Since the infection fatality risk (IFR) of SARS-CoV-2 infection in healthy 18-30 year old individuals (e.g., those who might plausibly be eligible to volunteer for HCS) is likely around 0.0070.03 per cent to begin with (Verity et al. 2020; Salje et al. 2020), marginal risk in locations with significant background risk would be a fraction of this (i.e., extremely low). An estimate of marginal fatality risk can be determined by multiplying the IFR by the probability 
of in-study infection (which might be reduced from 90100 per cent to $50-65$ per cent in a 1:1 randomized HCS, depending on the degree to which the vaccine being tested prevents infection) and subtracting the background risk of infection (which might be low in the short term under public health lockdown but potentially greater than 50 per cent in the longer term in communities with prolonged/recurrent high incidence epidemics) (Heriot et al. 2020; Salje et al. 2020). Under certain conditions, an individual's marginal risk (if there is significant background risk and/or significant vaccine efficacy in the trial) might range from half the IFR to zero or less (i.e. a net benefit of participation). The relative marginal risk reduction due to background risk would be high during high incidence epidemics, although the absolute risk reduction might be low because risks of infection for young healthy adults are small in any case. Still, because rare severe outcomes may nevertheless occur, and because public trust might depend on challenge trials being conducted to especially high standards, risk minimization remains an essential part of study design (WHO Working Group for Guidance on Human Challenge Studies in COVID-19 2020).

\section{Conclusions}

Ethical assessments of the benefits and risks of SARSCoV-2 HCS should be particularly systematic and must take into consideration the pandemic setting in which these studies might occur. Assessments should include a thorough evaluation of the potential public health benefits and marginal risks of HCS as compared to vaccine field trials or other methods of vaccine development. This will help to ensure that informed judgements are madeincluding decisions about trade-offs between different ethical considerations, which may vary between settings. The justification of SARS-CoV-2 HCS might therefore be context specific, and local decision-makers should be informed by the best available evidence including modelling of the benefits and risks of possible research strategies under different epidemiologic conditions.

\section{References}

Bambery, B., M. Selgelid, C. Weijer, J. Savulescu, and A.J. Pollard. 2015. Ethical criteria for human challenge studies in infectious diseases. Public Health Ethics 9(1): 92-103.
Bandaranayake, D., A. Bissielo, S. Huang, and T. Wood. 2010. Seroprevalence of the 2009 influenza A (H1N1) pandemic in New Zealand. Institute of Environemntal Science and Research Limited, National Centre for Biosecurity and Infectious Diseases, Client Report FW10057. https://www.health.govt. nz/system/files/documents/publications/seroprevalence-flu2009.pdf. Accessed August 10, 2020.

Callow, K., H. Parry, M. Sergeant, and D. Tyrrell. 1990. The time course of the immune response to experimental coronavirus infection of man. Epidemiology \& Infection 105(2): 435-446.

Cohen, J. 2020. Speed coronavirus vaccine testing by deliberately infecting volunteers? Not so fast, some scientists warn. Science Magazine, March 31. https://www.sciencemag. org/news/2020/03/speed-coronavirus-vaccine-testingdeliberately-infecting-volunteers-not-so-fast-some. Accessed August 10, 2020.

Eyal, N., and M. Lipsitch. 2020. Ethical comparators in coronavirus vaccine trials. http://nrs.harvard.edu/urn-3:HUL. InstRepos:42643064. Accessed August 10, 2020.

Eyal, N., M. Lipsitch, and P.G. Smith. 2020. Human challenge studies to accelerate coronavirus vaccine licensure. Journal of Infectious Diseases 221(11): 1752-1756.

Gates, B. 2020. Responding to Covid-19-A once-in-a-century pandemic? New England Journal of Medicine 382(18): 1677-1679.

Heriot, G.S., E. Jamrozik, and M.J. Selgelid. 2020. Tensions between public health and vaccine research priorities: A comparative modelling assessment of the risks and benefits of SARS-CoV-2 vaccine field trials versus human challenge studies.

Hilding, D.A. 1994. Literature review: The common cold. Ear, Nose \& Throat Journal 73(9): 639-647.

Hope, T., and J. McMillan. 2004. Challenge studies of human volunteers: Ethical issues. Journal of Medical Ethics 30(1): 110-116.

Jamrozik, E., and M.J. Selgelid. 2020a. Human challenge studies in endemic settings: Ethical and regulatory issues. Springer International Publishing.

- 2020b. COVID19 human challenge studies: Ethical issues. Lancet Infectious Diseases 20(8): e198-e203.

. 2020c. Ethical issues surrounding controlled human infection challenge studies in endemic low-and middle-income countries. Bioethics.

Jin, C., M.M. Gibani, M. Moore, et al. 2017. Efficacy and immunogenicity of a Vi-tetanus toxoid conjugate vaccine in the prevention of typhoid fever using a controlled human infection model of Salmonella Typhi: A randomised controlled, phase $2 \mathrm{~b}$ trial. The Lancet 390(10111): 2472-2480.

Kahn, R., M. Hitchings, R. Wang, S.E. Bellan, and M. Lipsitch. 2019. Analyzing vaccine trials in epidemics with mild and asymptomatic infection. American Journal of Epidemiology 188(2): 467-474.

Krzyzanowski, M., D.L. Sherrill, and M.D. Lebowitz. 1990. Longitudinal analysis of the effects of acute lower respiratory illnesses on pulmonary function in an adult population. American Journal of Epidemiology 131(3): 412-422.

Lederer, S.E. 2008. Walter Reed and the yellow fever experiments. In The Oxford textbook of clinical research ethics, edited by E.J. Emanuel, C.C. Grady, R.A. Crouch et al., 9-17. USA: Oxford University Press. 
Lipsitch, M., R. Kahn, and M.J. Mina. 2020. Antibody testing will enhance the power and accuracy of COVID-19-prevention trials. Nature Medicine 26: 818-819.

Lurie, N., M. Saville, R. Hatchett, and J. Halton. 2020. Developing COVID-19 vaccines at pandemic speed. New England Journal of Medicine 382(21): 1969-1973.

Memoli, M.J., P.A. Shaw, A. Han, et al. 2016. Evaluation of antihemagglutinin and antineuraminidase antibodies as correlates of protection in an influenza $\mathrm{A} / \mathrm{H} 1 \mathrm{~N} 1$ virus healthy human challenge model. MBio 7(2): e00417-e00416.

National Health and Medical Research Council and Australian Research Council. 2018. National Statement on Ethical Conduct in Human Research. Canberra: Commonwealth of Australia.

Phelan, A.L. 2020. COVID-19 immunity passports and vaccination certificates: scientific, equitable, and legal challenges. The Lancet 395(10237): 1595-1598.

Plotkin, S.A., and A. Caplan. 2020. Extraordinary diseases require extraordinary solutions. Vaccine 38(24): 3987.

Salje, H., C.T. Kiem, N. Lefrancq, et al. 2020. Estimating the burden of SARS-CoV-2 in France. Science 369(6500): 208211.

Schaefer, G., C.C. Tam, J. Savulescu, and T.C. Voo. 2020. Covid19 vaccine development: Time to consider Sars-Cov-2 challenge studies? Doi: https://doi.org/10.2139/ssrn.3568981.

Shah, S.K., J. Kimmelman, A.D. Lyerly, et al. 2017. Ethical considerations for Zika virus human challenge trials. National Institute of Allergy and Infectious Diseases.
Tacket, C.O., M.B. Cohen, S.S. Wasserman, et al. 1999. Randomized, double-blind, placebo-controlled, multicentered trial of the efficacy of a single dose of live oral cholera vaccine CVD 103-HgR in preventing cholera following challenge with Vibrio cholerae $\mathrm{O} 1 \mathrm{El}$ tor inaba three months after vaccination. Infection and immunity. 67(12): 6341-6345.

Vannice, K.S., M.C. Cassetti, R.W. Eisinger, et al. 2019. Demonstrating vaccine effectiveness during a waning epidemic: A WHO/NIH meeting report on approaches to development and licensure of Zika vaccine candidates. Vaccine 37(6): 863-868.

Verity, R., L.C. Okell, I. Dorigatti, et al. 2020. Estimates of the severity of coronavirus disease 2019: A model-based analysis. The Lancet Infectious Diseases 20(6): 669-677.

Wenner, D.M. 2018. Exploitation and international clinical research: The disconnect Between goals and policy. The Palgrave Handbook of Philosophy and Public Policy, edited by D. Boonin, 563-574. Palgrave Macmillan and Springer Nature.

WHO Working Group for Guidance on Human Challenge Studies in COVID-19 2020. Key criteria for the ethical acceptability of COVID-19 human challenge studies. Geneva, Switzerland.

Publisher's note Springer Nature remains neutral with regard to jurisdictional claims in published maps and institutional affiliations. 